\title{
Forebrain Glucocorticoid Receptors Modulate Anxiety-Associated Locomotor Activation and Adrenal Responsiveness
}

\author{
Maureen P. Boyle, ${ }^{1}$ Benedict J. Kolber, ${ }^{1}$ Sherri K. Vogt, ${ }^{1}$ David F. Wozniak, ${ }^{2}$ and Louis J. Muglia ${ }^{1}$ \\ Departments of ${ }^{1}$ Pediatrics and Molecular Biology and Pharmacology and ${ }^{2}$ Psychiatry, Washington University, St. Louis, Missouri 63110
}

\begin{abstract}
Stress potently modulates anxiety- and depression-related behaviors. In response to stressors, the hypothalamic-pituitary-adrenal (HPA) axis is activated, resulting in the release of glucocorticoids from the adrenal cortex. These hormones act peripherally to restore homeostasis but also feed back to the CNS to control the intensity and duration of the stress response. Glucocorticoids act in limbic areas of the CNS to mediate the psychological and behavioral effects of stress. In this study, we investigate the effect of forebrain-specific disruption of the glucocorticoid receptor (GR) on stress- and anxiety-related behaviors. We demonstrate that mice with disruption of forebrain GR show alterations in stress-induced locomotor activation in a number of anxiety-related behavioral paradigms. These changes are associated with alterations in stress-induced HPA axis activation and, importantly, are not attenuated by chronic treatment with the tricyclic antidepressant imipramine. These data demonstrate the importance of forebrain GR in regulation of physiological and behavioral stress reactivity and suggest that distinct pathways regulate despair- and anxiety-related behaviors.
\end{abstract}

Key words: glucocorticoid receptor; HPA axis; anxiety; knock-out mice; behavior; forebrain

\section{Introduction}

Appropriate regulation of the hypothalamic-pituitary-adrenal (HPA) axis is essential for health and survival (Bjorntorp and Rosmond, 2000; Chrousos, 2000). HPA axis activation and inhibition are complex processes, involving structures throughout the CNS (Laugero, 2001). Information regarding physiologic threats (i.e., hypothermia, hypoxia, and hypoglycemia) relay from the brainstem through the paraventricular nucleus (PVN) and anterior pituitary, leading to a prompt HPA axis response. Psychological stressors, or perceived threats, relay through limbic forebrain circuits. These circuits involve multiple brain regions, including the hippocampus, amygdala, cortex, locus ceruleus, bed nucleus of the stria terminals, and the nucleus of the tractus solitarius (Herman et al., 2003). The intricacy of these circuits allow for a diversity of stressor-specific responses (Herman et al., 2003). Moreover, disruption of forebrain/limbic circuits has been proposed to be involved in the development of affective and anxiety disorders (Coryell et al., 1989; Holsboer, 2000; Ehlert et al., 2001).

Anxiety disorders are often associated with hyperactivity of the HPA axis (Holsboer, 2000). These HPA axis changes strongly correlate with psychiatric disease states, because recovery is associated with reversal of the HPA axis abnormality. Although the

Received May 28, 2005; revised Jan. 4, 2006; accepted Jan. 7, 2006.

This work was supported by National Institutes of Health Grants AG18876 (L.J.M.) and MH067374-02 (M.P.B.) We thank Drs. James Herman, Lisa Muglia, and Alana Conti for manuscript review and helpful discussions and Lauren Kolber for graphical assistance.

Correspondence should be addressed to Dr. Louis J. Muglia, Washington University School of Medicine, 660 South Euclid Avenue, Box 8208, St. Louis, M0 63110. E-mail: muglia_@@kids.wustl.edu.

DOI:10.1523/JNEUROSCI.2173-05.2006

Copyright $\odot 2006$ Society for Neuroscience $\quad$ 0270-6474/06/261971-08\$15.00/0 importance of HPA axis dysregulation in affective disorders is recognized, the specific role that different brain regions play in regulation of the HPA axis and how these different regions contribute to anxiety-related disorders remain unknown.

The HPA axis is activated in a circadian rhythm and in response to stress. Activation begins with the release of corticotropin-releasing hormone $(\mathrm{CRH})$ and arginine vasopressin (AVP) from the PVN of the hypothalamus. These factors induce the release of adrenocorticotropin-releasing hormone (ACTH) from the anterior pituitary. ACTH travels through the bloodstream stimulating secretion of glucocorticoids [cortisol in humans, corticosterone (CORT) in rodents] from the adrenals. Glucocorticoids bind to glucocorticoid receptors (GR) to mediate the peripheral stress response and also feed back to the CNS to modulate further activation of the HPA axis (Herman et al., 2003).

The CNS expresses two glucocorticoid-responsive receptors with distinct but overlapping patterns of expression: type I, or mineralocorticoid receptors (MR), and type II, or GR (De Kloet et al., 1998). Because of the low abundance and the high affinity of MR for glucocorticoids, these receptors are believed to be mostly occupied at basal circadian glucocorticoid levels (Oitzl et al., 1995). GR become occupied as stress/circadian CORT release increases, mediating behavioral activation and providing negative feedback inhibition to the HPA axis (De Kloet et al., 1998; Kellendonk et al., 2002).

A number of findings have implicated forebrain GR in the regulation of the HPA axis and the development of affective disorders (DeRijk and Sternberg, 1997; Invitti et al., 1999; Holsboer, 2000). Our recent studies have described the production of knock-out mice with conditional disruption of GR in the fore- 
brain (FBGRKO) that show increased depression-related behavior and impairments in negative feedback regulation of the HPA axis (Boyle et al., 2005). In these mice, GR is disrupted in the hippocampus and cortex but not in the central nucleus of the amygdala, PVN, or pituitary. FBGRKO mice begin to delete GR at $\sim 3$ weeks of age, eliminating confounds associated with developmental effects of the loss of GR.

We hypothesize that a primary defect in forebrain GR will alter both behavioral and physiological responses to stress. In this study, we investigate how loss of forebrain GR influences anxietyrelated behaviors and stress-mediated regulation of the HPA axis. Our findings demonstrate that GR activity in the forebrain dynamically influences reactivity to stress.

\section{Materials and Methods Animal husbandry}

All mouse protocols were in accordance with National Institutes of Health guidelines and were approved by the Animal Care and Use Committee of Washington University School of Medicine (St. Louis, MO). Mice were housed on a $12 \mathrm{~h}$ light/dark cycle with ad libitum access to rodent chow and water.

\section{Generation of FBGRKO}

Mice homozygous for the previously described GRloxPneo allele (Brewer et al., 2003; Boyle et al., 2005) were crossed with mice expressing a transgene in which Cre recombinase is expressed under the control of the forebrain-specific calcium/calmodulin-dependent kinase II promoter (T50 Cre) (Tsien et al., 1996; Boyle et al., 2005). Mice homozygous for the GRloxPneo allele expressing Cre recombinase result in forebrain-specific disruption of GR (FBGRKO). Four- to 6-month-old male littermate knock-out and control (Cre negative) mice were used for all experiments. Mice were of a mixed C57BL/ $6 \times 129 \times$ CBA background.

\section{Immunohistochemistry}

FBGRKO and littermate controls were anesthetized with 2.5\% Avertin (intraperitoneally) and perfused transcardially with cold PBS, followed by cold $0.1 \mathrm{~m}$ PBS containing $4 \%$ paraformaldehyde. Brains were then embedded in paraffin, and $8-\mu \mathrm{m}$-coronal sections were collected. Sections were processed for immunohistochemistry with antibodies recognizing GR (1:200, M-20; Santa Cruz Biotechnology, Santa Cruz, CA) and neuronal-specific nuclear protein (NeuN) (1:200; Chemicon, Temecula, CA). Nonspecific binding for GR/NeuN was blocked with 3\% normal goat serum in PBS. Sections were incubated with primary antibody overnight at $4^{\circ} \mathrm{C}$, washed with PBS, incubated with secondary antibody for $1 \mathrm{~h}$ at room temperature [NeuN, 1:200 FITC-conjugated goat anti-mouse IgG (Jackson ImmunoResearch, West Grove, PA); GR, 1:250 biotinylated goat anti-rabbit IgG (Vector Laboratories, Burlingame, CA)], incubated in an avidin/biotin complex reagent (Vector Laboratories) for $1 \mathrm{~h}$, washed with PBS, and incubated in a cyanine-3-conjugated tyramide signal amplification reagent (PerkinElmer, Boston, MA).

\section{Behavioral analysis}

All behavioral analyses were performed between 8 A.M. and 12:00 P.M. by an observer blinded to genotype.

Light/dark preference. A two-compartment standard shuttle box $(20.3 \times 15.9 \times 21.3 \mathrm{~cm}$; Med Associates, St. Albans, VT) with two communicating compartments of equal size and a stainless steel bar floor was used. The compartments are separated by a $3 \times 4 \mathrm{~cm}$ sliding door built into the separating wall. Mice were placed in the dark compartment and allowed to acclimate for $1 \mathrm{~min}$. The slide door was then opened, and the latency to enter the light compartment, the total time spent in the light compartment, and the total number of entries into the light compartment were recorded for a $10 \mathrm{~min}$ trial. In a control experiment, mice were placed in the light compartment and allowed to acclimate for $1 \mathrm{~min}$. The slide door was opened, and the latency to enter the dark compartment was recorded.

Antidepressant treatment. FBGRKO and control mice were treated with imipramine $(16 \mathrm{mg} / \mathrm{kg}$ in $0.9 \%$ normal saline, $\mathrm{pH} 7.5)$ or vehicle. Mice were injected intraperitoneally daily (between 9:00 A.M. and 10:00
A.M.) for 3 weeks and tested for light/dark preference $24 \mathrm{~h}$ after their last dose.

Elevated plus maze. Our apparatus consisted of two opposing open arms and two opposing enclosed arms $(35 \times 6.1 \times 15 \mathrm{~cm})$ that extended from a central platform $(5.5 \times 5.5 \mathrm{~cm})$. The floor and walls of the elevated plus maze (EPM) were constructed of black Plexiglas. The maze was equipped with pairs of photocells configured in a 16 ( $x$-axis $) \times 16$ ( $y$-axis) matrix, the output of which was recorded by a computer and interface assembly (Hamilton-Kinder, Poway, CA). A software program (Hamilton-Kinder) enables the beam-break data to be recorded and analyzed to quantify time spent, distance traveled, time spent at rest, and the numbers of entries made into the open and closed arms and the center area. The numbers of fine movements and $x$ - and $y$-axes ambulations were also recorded throughout the maze. Test sessions commenced by placing a mouse in the center of the maze, allowing the mouse to explore the maze. Each test session lasted $5 \mathrm{~min}$, and mice were tested over 3 consecutive days.

Forced swim test. Mice were placed in a $2 \mathrm{~L}$ beaker half-filled with water $\left(18-20^{\circ} \mathrm{C}\right)$. The level of the water prevented the animals from escaping or from reaching the bottom of the container. Every $5 \mathrm{~s}$ during a $15 \mathrm{~min}$ trial, an observer recorded the activity of the mouse. If the mouse was active at any time during a given $5 \mathrm{~s}$ bin, $\mathrm{a}+1$ was recorded, and, if the animal remained inactive throughout the entire $5 \mathrm{~s}$ bin, a 0 was recorded. At the end of the trial, the animal was removed from the water, dried, and returned to its home cage. Graphs were generated by calculating the percentage of bins that each mouse was active during the trial.

Open-field behavior. Our open-field apparatus consisted of a Plexiglas box ( 2.5 feet wide, 2.5 feet long, 1 foot height). A 5 square $\times 5$ square (each square with 0.5 feet sides) grid was outlined on the surface of the arena, with the outer rim labeled quadrant 3 , the inner area labeled quadrant 2 , and the center square labeled quadrant 1 . Each mouse was placed in a corner of the open field under standard light conditions. The experiment lasted for $10 \mathrm{~min}$. Between sessions, the maze was rinsed with $70 \%$ ethanol and dried with paper towels. Activity was observed, and the time spent in each of the three quadrants and the latency to enter quadrants 2 and 1 were recorded.

\section{In situ hybridization}

Brains were collected under basal conditions or $1 \mathrm{~h}$ after the initiation of either mild or restraint stress. Mice were deeply anesthetized with $1 \mathrm{ml}$ of $2.5 \%$ Avertin and then transcardially perfused with diethylpyrocarbonate (DEPC)-treated PBS, followed by $4 \%$ DEPC paraformaldehyde. Isolated brains were postfixed in $4 \%$ paraformaldehyde for $24 \mathrm{~h}$, followed by immersion in $10 \%$ sucrose in DEPC PBS. Tissues embedded in OCT (Sakura Finetek USA, Torrance, CA) were cut into $15 \mu \mathrm{m}$ sections on a cryostat and thaw mounted onto Superfrost plus slides (Fisher Scientific, Pittsburgh, PA). RNA probes complementary to mRNA for MR, CRH, $\mathrm{AVP}$, or CRH receptor 1 (CRHR1) were radiolabeled with $\alpha^{33} \mathrm{UTP}$, hybridized to sections at an annealing temperature of $60^{\circ} \mathrm{C}$, and washed, after hybridization, in $0.1 \times \mathrm{SSC}$ at $65^{\circ} \mathrm{C}$ for $30 \mathrm{~min}$. Slides were exposed for $6 \mathrm{~h}$ to $7 \mathrm{~d}$ to Hyperfilm $\beta$ Max (Amersham Biosciences, Arlington Heights, IL). Autoradiographic images were scanned at 2400 dots per inch on an Epson 1680 Pro scanner. Densitometric analysis of in situ signal was performed using NIH Image software.

\section{Radioimmunoassays}

Plasma concentrations of corticosterone was determined by RIA (ICN Pharmaceuticals, Costa Mesa, CA; DiaSorin, Stillwater, MN) from blood collected by retro-orbital phlebotomy from adult male mice as described previously (Boyle et al., 2005).

Restraint stress. Restraint was performed in a ventilated $50 \mathrm{ml}$ centrifuge tube for 5,15 , or $30 \mathrm{~min}$, after which plasma was immediately collected. Plasma was also collected at 30, 60, and 90 min after the termination of $30 \mathrm{~min}$ of restraint stress. Each group consisted of 8-10 mice.

Mild stress. Mild stress consisted of an intraperitoneal needle stick with a sterile 18 gauge needle. Plasma was collected 5, 15, 30, 60, 90, and 120 min after the needle stick. 


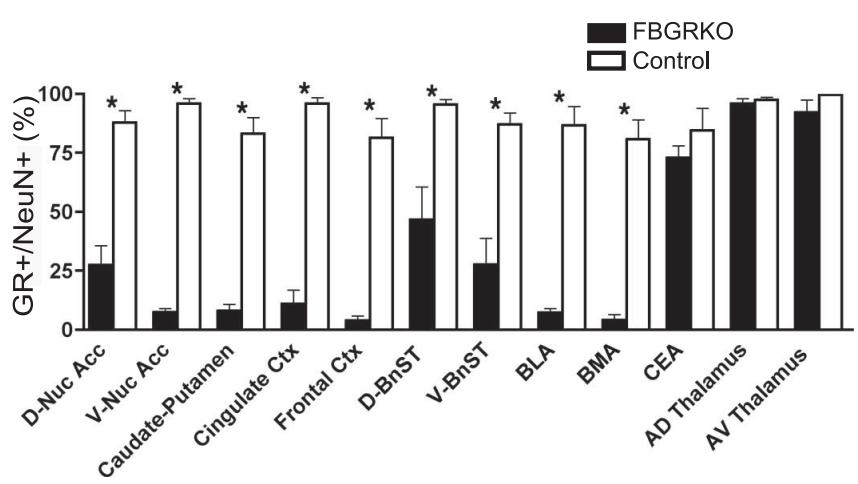

Figure 1. Region-specific disruption of neuronal GR expression in FBGRKO mice. Neuronal GR expression was evaluated by colocalization of GR and NeuN immunoreactivity in the dorsal and ventral nucleus accumbens (D-Nuc Acc, V-Nuc Acc), caudate-putamen, cingulate cortex (Ctx), frontal cortex, dorsal and ventral bed nucleus of the stria terminalis (D-BnST, V-BnST), basolateral amygdala (BLA), basomedial amygdala (BMA), central nucleus of the amygdala (CEA), and anterodorsal (AD) and anteroventral (AV) thalamic nuclei ( $n=3-4$ mice per genotype, $2-4$ sections per animal per region; ${ }^{*} p<0.05$ control vs FBGRKO in specific region).

\section{ACTH stimulation test}

Cortrosyn (Organon Pharmaceuticals, West Orange, NJ) was reconstituted in $1 \mathrm{ml}$ of normal saline. The Cortrosyn solution was then diluted 1:250 in PBS, $50 \mu \mathrm{g} / \mathrm{kg}$ was injected intraperitoneally, and blood was collected 45 min later.

\section{Statistical analysis}

Results were expressed as mean \pm SEM. Statistical comparisons were performed with the use of one- or two-way ANOVA with the post hoc Student-Newman-Keuls test to identify significant differences. In all cases, $p<0.05$ was considered statistically significant.

\section{Results}

\section{Region-specific disruption in FBGRKO mice}

To define the extent of GR disruption in different forebrain regions implicated in physiological and behavioral responses to stress, we quantified the number of neurons displaying GR immunoreactivity in 4- to 6-month-old FBGRKO and control mice by performing GR and NeuN double-labeling experiments. Extensive loss of GR immunoreactivity occurred in neurons throughout the cerebral cortex, nucleus accumbens, and caudate-putamen in the FBGRKO mice (Fig. 1). GR immunoreactivity in the central nucleus of the amygdala was preserved, consistent with our previous report (Boyle et al., 2005). In contrast, the basolateral and basomedial amygdala demonstrated disruption of GR expression in the FBGRKO mice (supplemental Fig. S1, available at www.jneurosci.org as supplemental material). Partial loss of GR expression was found in the bed nucleus of the stria terminalis. As expected, GR expression was not altered in the thalamus of FBGRKO mice.

\section{Altered regulation of anxiety-related behaviors in FBGRKO mice}

FBGRKO mice exhibit increased depression-like behavior (Boyle et al., 2005). Controversy exists as to whether anxiety and depression represent distinct disorders or different behavioral outputs resulting from the same underlying dysfunction (Gorman, 1996; Barlow and Campbell, 2000). We, therefore, examined anxietyrelated behaviors in FBGRKO and control mice. Light/dark preference is a commonly used measure of anxiety-like behavior in rodents. The inherent preference of the mouse to be in the dark chamber (away from environmental threats) offsets the desire to explore a new environment. Increased anxiety-related behavior is reflected by increased time spent in the dark compartment. Naive

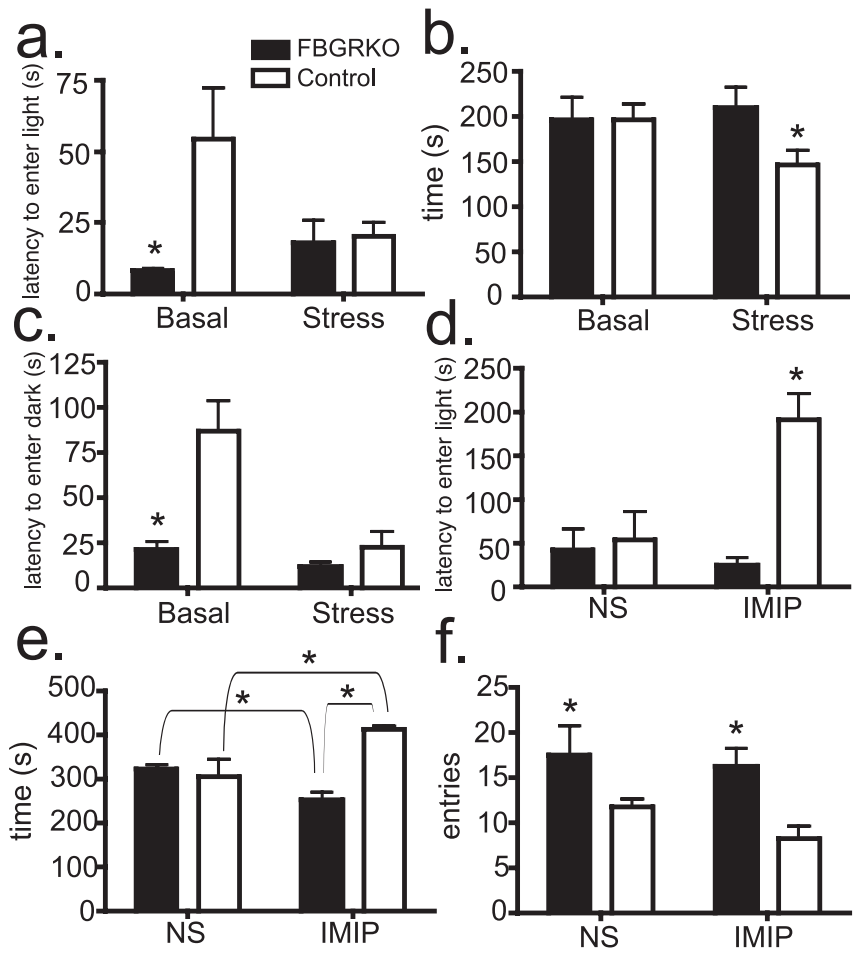

Figure 2. Altered stress reactivity in FBGRK0 mice. $\boldsymbol{a}$, In the light/dark preference test $(n=$ 5-6), FBGRKO mice show a significantly decreased latency to enter the light compartment $\left({ }^{*} p=0.03\right)$ compared with controls. Restraint stress significantly reduced the latency to enter the light compartment in controls but not in FBGRKO mice $(p=0.03) . \boldsymbol{b}$, No basal differences were found between genotypes in time spent in the light compartment. After stress, control mice spent significantly less time in the light compartment $\left({ }^{*} p=0.05\right)$. Stress had no effect on this measure in FBGRKO mice. c, In a modified version of the light/dark preference test, mice were placed in the light compartment, and the time to enter the dark compartment was measured. In the unstressed condition, FBGRKO mice show a significantly reduced latency to enter the dark chamber $\left({ }^{*} p=0.001\right)$. Restraint stress significantly reduced the latency to enter the light compartment in controls but not in FBGRKO mice $\left({ }^{*} p=0.003\right)$. $\boldsymbol{d}$, The chronic mild stress associated with daily normal saline (NS) injections resulted in no difference in latency to enter the light compartment between genotypes. Treatment with imipramine (IMIP) produced a significant increase in this measure in control mice $\left({ }^{*} p=0.03\right)$. e, Imipramine treatment $(n=$ 4) led to a significant increase in time spent in the light compartment in control mice ( $p=0.04)$ and a significant decrease in this measure in FBGRKO mice $(p=0.01)$. The net result produced a significant difference between genotypes ( $p<0.0001)$. $f$, FBGRKO mice show a significantly increased $\left(^{*}\right)$ number of entries into the light compartment compared with controls when treated with either normal saline ( $p=0.04$ ) or imipramine ( $p=0.02$ ).

FBGRKO mice show decreased latency to enter the light compartment compared with controls (Fig. $2 a)(p=0.03 ; n=5-6)$. No basal differences were found in time spent in the light compartment across genotypes (Fig. $2 b$ ). After $30 \mathrm{~min}$ of restraint stress, control mice showed the expected decrease in time spent in the light chamber (Fig. $2 b)(p=0.05)$ and a significant decrease in latency to enter the light compartment (Fig. $2 a)(p=0.03)$ compared with basal. In contrast, FBGRKO mice showed no changes in latency to enter or time in the light chamber after restraint stress.

Mice were also tested in a version of the light/dark preference test in which they were placed in the light compartment and the latency to enter the dark compartment was recorded. FBGRKO mice showed a decreased latency to enter the dark compartment relative to controls (Fig. $2 c)$ ( $p=0.001$ ). After $30 \mathrm{~min}$ of restraint stress, FBGRKO mice showed no change, whereas control mice showed a significant decrease in latency to enter the dark compartment compared with basal $(p=0.003)$. 
a.
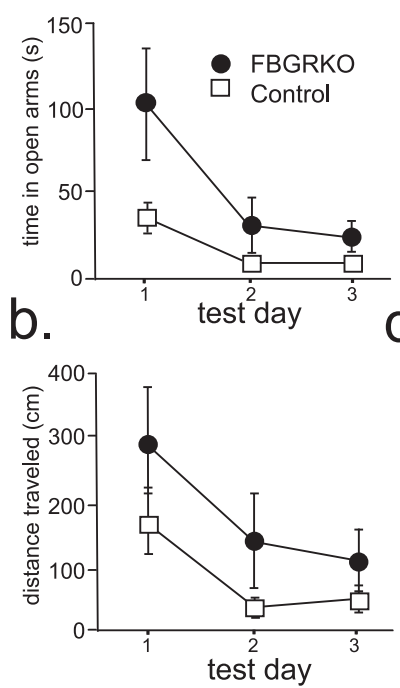

C.
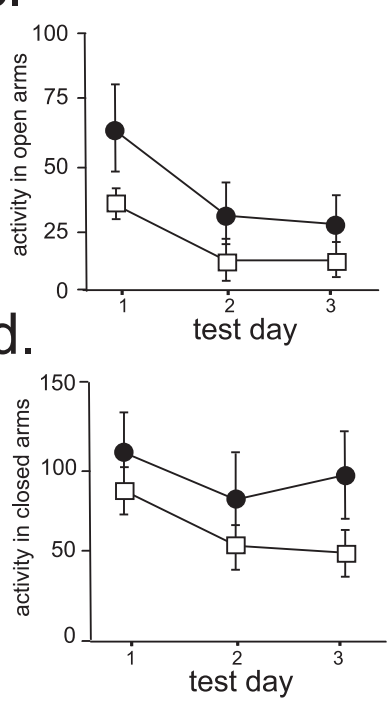

Figure 3. Anxiety-related behaviors in FBGRKO and control mice. Analysis of behavior in the elevated plus maze $(n=8-9)$ revealed that FBGRKO mice spend significantly more time ( $p=$ $0.03)(\boldsymbol{a})$ and travel significantly more distance $(p=0.05)(\boldsymbol{b})$ in the open arms. This change was associated with a significant increase in locomotor activity in both the open ( $p=0.01)$ (c) and closed ( $p=0.02)(\boldsymbol{d})$ arms of the maze.

Reduced anxiety-like behavior in control but not FBGRKO mice after chronic treatment with imipramine

Mice treated with the tricyclic antidepressant imipramine for 3 weeks were tested in the light/dark preference test $(n=5)$. Similar to our results after restraint stress, control mice treated daily with normal saline, equivalent to a chronic mild stress, showed an equivalent latency to enter the light compartment as salinetreated FBGRKO mice. Interestingly, imipramine treatment in the control mice led to increased latency to enter the light compartment when compared with FBGRKO mice (Fig. $2 d)(p=$ $0.03)$. Chronic imipramine treatment had no effect on latency to enter the light compartment in FBGRKO mice. Imipramine treatment also increased the time spent in the light chamber in control mice (Fig. 2e) $(p=0.04)$, suggesting a decrease in anxiety-related behavior. FBGRKO mice treated with imipramine, however, spent somewhat less time in the light compartment compared with mice treated with normal saline $(p=0.04)$. FBGRKO mice showed a significantly greater number of entries into the light compartment compared with controls when treated with imipramine (Fig. $2 f)(p=0.04)$ or normal saline $(p=$ $0.02)$.

\section{Locomotor activation in the elevated plus maze}

The EPM is one of the most commonly used tests of anxiety-like behavior in rodent models. The FBGRKO mice spent significantly more time in the open arms (Fig. $3 a)(p=0.03)$ and traveled significantly greater distance in the open arms (Fig. $3 b$ ) $(p=0.05)$ compared with control mice. These alterations were associated with increased locomotor activity in both the open (Fig. $3 c)(p=0.01)$ and closed arms (Fig. $3 d)(p=0.02)$.

We also evaluated the behavior of FBGRKO and control mice on a second test commonly used for the measurement of innate anxiety-like behaviors, the open-field test. No differences were found in anxiety-like behaviors (Fig. 4a) or activity (Fig. $4 b$ ) between FBGRKO and control mice in this test $(n=10)$. The similar activity between control and FBGRKO mice in this open-field

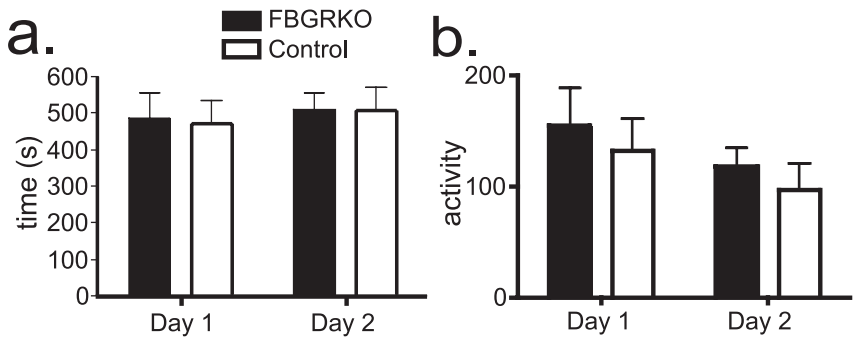

Figure 4. No differences in open-field behavior in FBGRKO mice. $\boldsymbol{a}$, FBGRKO and control mice took an equivalent amount of time to enter the center of the open field (quadrant 1) on both days $(n=10)$. Similar results were found for time to enter quadrant 2 (data not shown). $\boldsymbol{b}$, No differences were found in the activity of FBGRKO and control mice during open-field testing as assessed by line crossing in the arena.

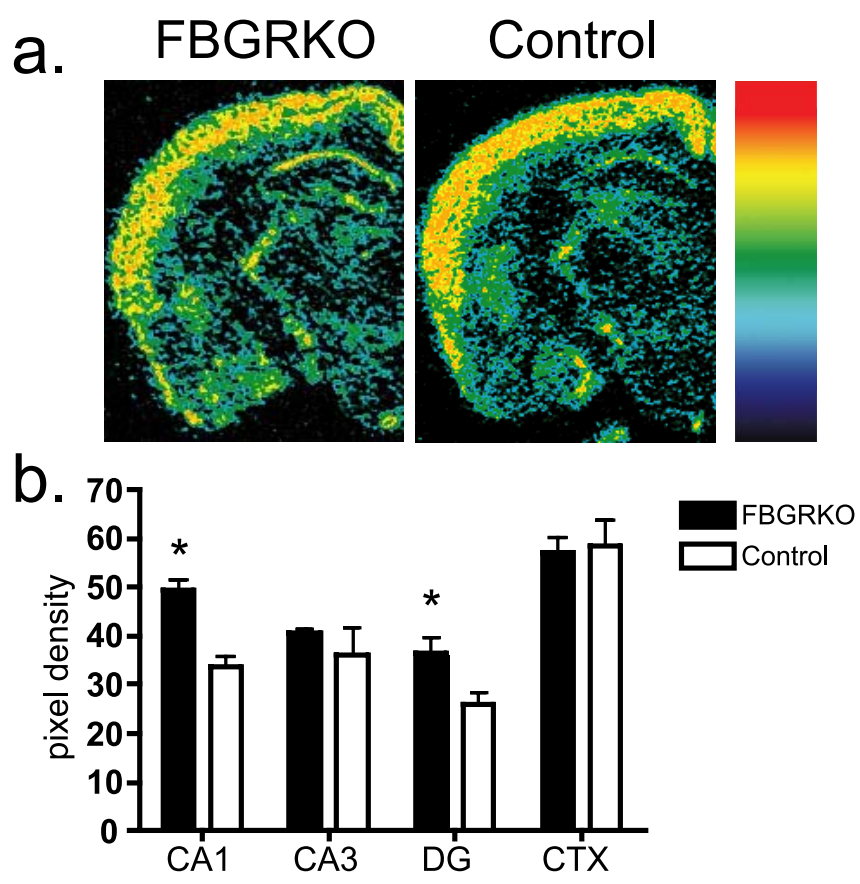

Figure 5. Increased hippocampal CRHR1 expression in FBGRKO mice. $\boldsymbol{a}$, Representative sections of pseudocolored in situ hybridization images from FBGRKO and control mice with the color gradient applied from low (black) to high (red). $\boldsymbol{b}$, Quantitative analysis of CRHR1 mRNA $(n=3-4)$ revealed significantly increased expression $\left({ }^{*}\right)$ in areas CA1 $(p=0.003)$ and the dentate gyrus (DG; $p=0.03$ ) of the hippocampus in FBGRKO mice. CTX, Cortex.

measure is consistent with our previous analysis of general locomotor activity under basal conditions using beam breaks in a photocell apparatus (Schaefer et al., 2000; Boyle et al., 2005). Thus, the FBGRKO mice are not hyperactive in general.

Alterations in anxiety-related behavior are associated with increased hippocampal CRHR1 expression in FBGRKO mice CRHR1 signaling regulates anxiety-related behaviors (Holsboer, 1999). Mice deficient for this receptor show decreased anxietylike behavior on a variety of behavioral tests (Muller et al., 2003). In addition, CRHR1 antagonists have been shown to be effective as anxiolytics and as antidepressants (Holsboer, 1999). We analyzed CRHR1 mRNA expression by in situ hybridization in FBGRKO and control mice $(n=3-4)$. FBGRKO mice show increased expression in area CA1 (Fig. 5) $(p=0.004)$ and the 


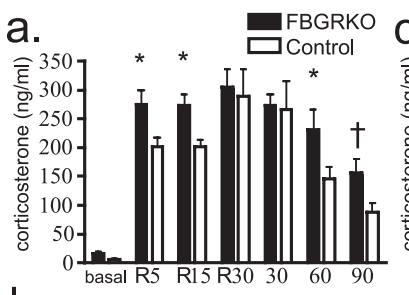

b.

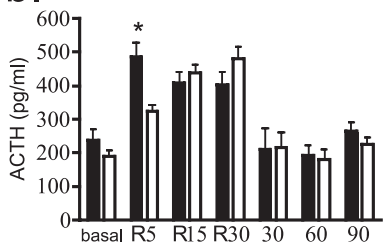

\begin{abstract}
d.
\end{abstract}
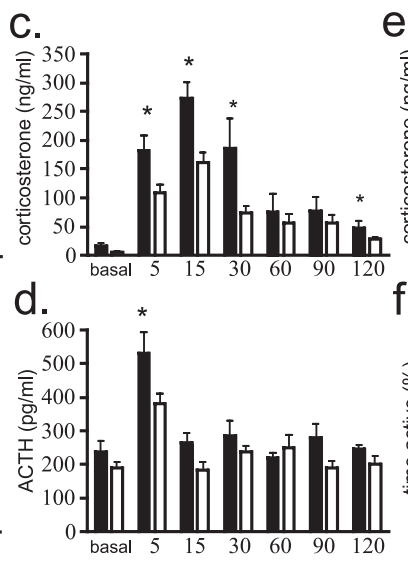

e.

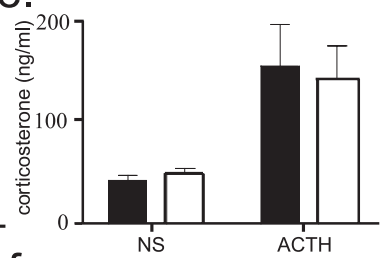

f.

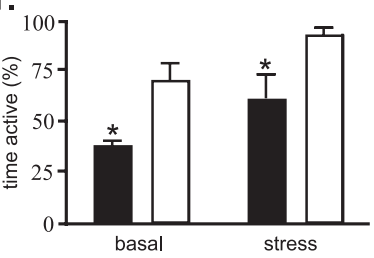

Figure 6. HPA axis regulation after stress. $\boldsymbol{a}$, Analysis of CORT secretion after restraint stress $(n=4-5)$ revealed that FBGRKO mice secrete significantly more $\left({ }^{*}\right)$ CORT after 5 and 15 min of restraint stress. Plasma CORT was also significantly higher in FBGRKO mice 60 min after the termination of restraint stress $(p=0.04)$, and a trend toward increased CORT secretion was found after 90 min in FBGRKO mice $(\dagger p=0.06)$. $\boldsymbol{b}$, FBGRKO mice secrete significantly more ACTH after 5 min of restraint stress compared with controls. c, FBGRKO mice secrete significantly more CORT 5, 15, and $30 \mathrm{~min}$ ( $p=0.05)$ but not 60 or $90 \mathrm{~min}$ after mild stress. Two hours after stress, FBGRKO mice again showed increased plasma CORT compared with controls $(p=0.05)$. $\boldsymbol{d}$, FBGRKO mice secrete significantly more ACTH 5 min after mild stress. $\boldsymbol{e}$, No differences were found between genotypes in the release of CORT after ACTH stimulation. NS, Normal saline. $f$, Analysis of activity in the forced swim test $(n=4-6)$ revealed that, after restraint stress, both genotypes show increased activity, but FBGRKO mice maintain decreased activity relative to controls ( $p=0.03$ ).

dentate gyrus $(p=0.03)$ of the hippocampus but not in area CA3 or the cortex.

\section{FBGRKO mice show altered kinetics of glucocorticoid release after stress}

To examine how disruption of forebrain GR influences HPA axis responses to stress, we subjected FBGRKO and control mice to either restraint stress or a brief, mild stress (needle stick; $n=$ 4-5). After 5 or 15 min of restraint, FBGRKO mice show significantly greater plasma concentrations of CORT compared with controls (Fig. 6a). After 30 min of restraint stress, FBGRKO and control mice release equivalent amounts of CORT, possibly representing a physiological maximum. Differences were also found in the time course of CORT decline after $30 \mathrm{~min}$ of restraint. These data suggest that the FBGRKO mice have an augmented CORT response and that these mice have impaired ability to terminate the stress response.

FBGRKO mice displayed significantly increased concentrations of plasma CORT $5 \mathrm{~min}(p=0.026), 15 \mathrm{~min}(p=0.008)$, and 30 min $(p=0.05)$ after the presentation of a mild stressor (needle stick) compared with controls (Fig. 6c). By 60 min, CORT release was equivalent across genotypes. A significant difference was also found at $2 \mathrm{~h}$ after stress $(p=0.05 ; n=4-5)$, likely reflecting the normal basal differences observed between these genotypes (Boyle et al., 2005).

\section{Increased ACTH secretion after stress in FBGRKO mice}

In their basal state, FBGRKO mice show a trend toward increased basal circadian ACTH release. After 5 min of restraint stress, both FBGRKO and control mice showed a significant increase in plasma ACTH concentrations (Fig. 6b). This increase was significantly greater in FBGRKO mice compared with controls ( $p=$ 0.021). After 15 or $30 \mathrm{~min}$ of restraint stress, ACTH levels increased in control mice, resulting in no significant difference from FBGRKO mice. By $30 \mathrm{~min}$ after the termination of $30 \mathrm{~min}$ of restraint stress, ACTH levels returned to near basal levels in both genotypes.

By 5 min after mild stress, FBGRKO mice released signifi- cantly more ACTH compared with controls (Fig. $6 d)(p=0.02)$. By 15 min after mild stress, ACTH levels approached basal concentrations in both genotypes.

No changes in ACTH-induced release of CORT in FBGRKO and control mice

To evaluate whether the increased CORT release after a mild stressor reflected an increased responsiveness to ACTH, we performed an ACTH stimulation test $(n=$ 6-7). We found no differences in ACTHinduced secretion of CORT in FBGRKO and control mice (Fig. 6e).

\section{Despair-like behaviors in FBGRKO mice after restraint stress}

We reported previously that naive FBGRKO mice show increased despair-like behaviors in the forced swim and tail suspension test, both standard measures of behavioral despair routinely used as a screen for rodent models of depression (Boyle et al., 2005). In this study, immediately after $30 \mathrm{~min}$ of restraint stress (a time at which plasma corticosterone levels are equivalent), we found that stress increased activity in both genotypes. FBGRKO mice, however, still maintained significantly reduced activity compared with stressed controls (Fig. $6 f)(p=0.03 ; n=6)$.

\section{Expression of CRH, AVP, and MR after restraint stress}

To examine the molecular correlates of altered HPA responsiveness to stress, we examined regulation of $\mathrm{CRH}, \mathrm{AVP}$, and MR after restraint and mild stress $(n=4)$. Analysis of CRH mRNA in the PVN showed no basal differences in FBGRKO and control mice (Boyle et al., 2005). CRH mRNA was found to be decreased in control mice $1 \mathrm{~h}$ after both types of stressors. In FBGRKO mice, CRH mRNA did not change after stress. The net result of these changes led to significantly more CRH mRNA in the PVN of FBGRKO mice after both mild $(p=0.05)$ and restraint $(p=$ 0.006) stress (Fig. 7a). Analysis of CRH mRNA expression in the amygdala, a site in which GR remains intact in FBGRKO mice, revealed no differences after mild stress. In contrast, restraint stress led to decreased expression in both genotypes (data not shown).

We next analyzed AVP mRNA expression in the PVN after mild and restraint stress. Similar to our previous findings, we found a basal increase in AVP mRNA expression in the PVN of FBGRKO mice compared with controls (Boyle et al., 2005). After mild stress, PVN expression of AVP mRNA increased slightly in controls but was unchanged in FBGRKO mice. After restraint stress, AVP expression was unchanged in FBGRKO and controls. AVP was significantly greater in FBGRKO mice in all conditions (Fig. 7b) $(p<0.05)$.

Under basal conditions, there were no differences in hippocampal MR expression in the FBGRKO and control mice (Fig. $7 c$ ). Mild stress led to an equivalent increase in MR expression in both groups across all hippocampal subfields (Fig. $7 d$ ). Interestingly, restraint stress produced no changes in MR expression in control mice but led to a significant decrease in levels of MR mRNA in area CA1 $(p=0.005)$ and the dentate gyrus $(p=0.01)$ in FBGRKO mice compared with controls (Fig. 7e). 


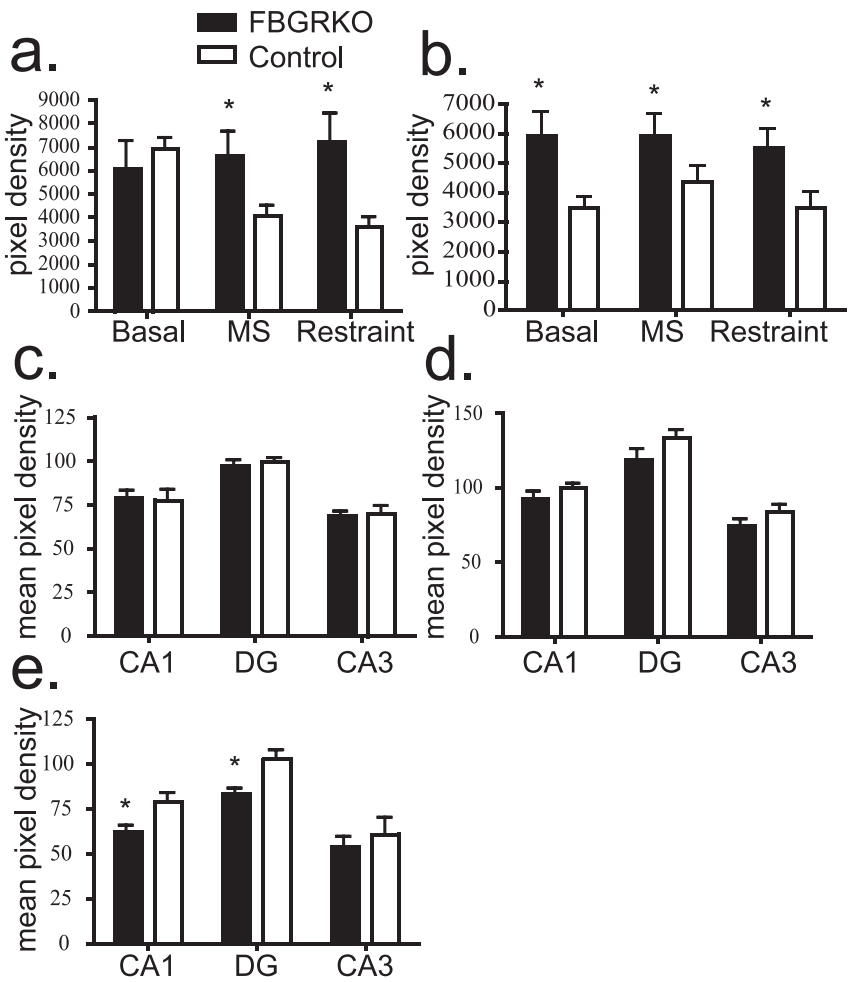

Figure 7. Expression of HPA axis regulators. $\boldsymbol{a}$, In the PVN, CRH mRNA expression is decreased after both mild (MS) $(p=0.05)$ and restraint stress $(p=0.006)$ in control but not FBGRKO mice. $\boldsymbol{b}$, AVP expression is significantly greater $\left({ }^{*}\right)$ in the PVN of FBGRKO mice basally ( $p=0.0014)$, after mild stress $(p=0.05)$, and after restraint stress $(p=0.04)$.c Analysis of hippocampal MR expression by in situ hybridization in naive FBGRKO and control mice revealed no differences across genotypes. $\boldsymbol{d}$, Analysis of MR expression after mild stress showed similar reductions in MR expression across hippocampal subfields between genotypes. $\boldsymbol{e}$, Analysis of MR expression after restraint stress revealed significantly reduced expression $(*)$ in hippocampal areas CA1 ( $p=0.006$ ) and the dentate gyrus ( $\mathrm{DG} ; p=0.012$ ) in the FBGRKO mice compared with stressed controls.

\section{Discussion}

Altered regulation of anxiety-like behaviors in FBGRKO mice after stress

Depression shows a high rate of comorbidity with anxiety disorders (Belzer and Schneier, 2004; Gorwood, 2004). It remains unclear, however, whether these diseases represent distinct disorders or different manifestations of the same underlying dysfunction (Tollefson et al., 1993). We therefore analyzed anxiety-related behaviors in FBGRKO and control mice. In the EPM, FBGRKO mice showed increased time spent and distance traveled in the open arms of the maze, as well as increased locomotor activity in both the open and closed arms. Increased time spent and distance traveled in the open arms of the EPM are typically thought to reflect an anxiolytic-like phenotype. Other observations, however, promote an alternate interpretation.

Evaluation of light/dark preference behaviors revealed a novel phenotype. FBGRKO mice enter the light compartment significantly earlier than controls, a pattern typically thought of as reflecting decreased anxiety-like behavior. However, control mice show decreased latency to enter the light compartment after acute (restraint) or chronic (daily intraperitoneal injection) stress. The effect of chronic stress was reversed after daily treatment with the antidepressant imipramine. When FBGRKO and control mice were initially placed in the light compartment and the latency to enter the dark compartment was recorded, FBGRKO mice showed a significant decrease in latency to enter the dark com- partment compared with controls. Similar to what we find in the light/dark preference test, restraint stress produced a significant decrease in latency to enter in the control mice. These data suggest that the phenotype in FBGRKO mice does not result from changes in anxiety-related behavior in light versus dark conditions. Accordingly, we interpret this measure to reflect that FBGRKO mice show increased locomotor activity or impulsivity in stressful situations. The acute stress response is commonly described as a "fight-or-flight" response. These data imply that FBGRKO mice demonstrate an exaggerated "flight" response compared with control mice. This interpretation is supported by the observation that FBGRKO mice made significantly more entries into the light compartment compared with controls. Increased locomotor activation or impulsivity in FBGRKO mice may also explain the phenotype observed in the elevated plus maze. The increased time spent and distance traveled in the open arms of the maze may be related to increased agitation in response to the stress associated with the test as opposed to reflecting decreased anxiety-like behavior. The lack of differences in anxiety-like behaviors or activity in the open-field test may result from the open-field test being a more natural environment that may not achieve a threshold stressful stimulus to distinguish genotypes.

In the light/dark preference test, time spent in the light compartment is the most commonly used measure for anxiety-like behavior. No differences were found in time spent in the light compartment in naive mice. Restraint stress, however, produced a decrease in time spent in the light compartment in control mice, reflecting increased anxiety-related behavior. In FBGRKO mice, however, restraint stress had no effect on this measure. After chronic treatment with imipramine, control mice showed a significant increase in the amount of time spent in the light chamber, suggesting that antidepressant treatment reduced anxietylike behavior in control mice. FBGRKO mice, conversely, showed a modest decrease in the amount of time spent in the light chamber after imipramine treatment. Thus, imipramine treatment was ineffective in reducing anxiety-related behavior in FBGRKO mice. We reported previously that chronic treatment with the same dose of imipramine reversed the circadian hyperactivity of the HPA axis and the behavioral despair-like phenotype in FBGRKO mice (Boyle et al., 2005). Together, our findings indicate that different pathways may mediate despair- and anxietylike behaviors. Furthermore, unlike despair-related behaviors, the failure of antidepressants to attenuate the anxiety-like behaviors suggests that GR could be a direct target for antianxiety effects.

\section{Altered despair-related behaviors are not attributable to differences in plasma corticosterone}

Naive FBGRKO mice show decreased activity in both the tail suspension and forced swim tests, indicating an increase in despair-related behaviors (Boyle et al., 2005). To examine the possibility that this difference may be secondary to the increase in circulating CORT, we examined these behaviors immediately after 30 min of restraint stress, when plasma CORT levels are equivalent. Although stress increased activity in both groups, FBGRKO mice still showed significantly less activity compared with controls. The increase in despair-like behaviors in the FBGRKO mice is therefore not likely to be attributable to acute differences in plasma corticosterone. 


\section{Alterations in post-stress regulation of the HPA axis in FBGRKO mice}

FBGRKO mice secrete significantly more CORT during restraint stress compared with controls. The CORT secretion reached higher levels earlier after restraint and decreased more slowly in the FBGRKO mice. At 30 min after restraint stress, both control and FBGRKO mice secrete equivalent amounts of CORT, perhaps reflecting a ceiling effect. Evaluation of CORT release at various time points after the termination of restraint stress revealed that FBGRKO mice turn off the stress response slower than controls, reflecting either impaired negative feedback inhibition of the HPA axis or a sustained increase in HPA axis drive from circuits stimulating hypothalamic activity. The finding that FBGRKO mice demonstrated a slower decrease in CORT is consistent with our previous observation of impaired glucocorticoidmediated suppression of the HPA axis after dexamethasone administration in the FBGRKO mice (Boyle et al., 2005). FBGRKO mice also release significantly more CORT compared with controls after a needle stick, indicating increased physiological reactivity to this mild stressor.

The observed increases in CORT secretion in FBGRKO mice occur in the context of increased acute ACTH secretion after both mild and restraint stress. Although FBGRKO ACTH levels returned to nonstressed levels in a manner similar to controls, these similar plasma ACTH concentrations occurred in the setting of higher circulating CORT levels in the FBGRKO mice. This finding suggests that the FBGRKO mice maintain a prolonged increase in CORT secretion by having a different set point for CORT modulation of hypothalamic-pituitary activity during stress.

\section{Changes in HPA axis regulation are correlated with altered regulation of CRH, CRHR1, and MR}

FBGRKO mice show increased AVP but not CRH mRNA expression in the PVN. In this study, we found that CRH expression in the PVN is decreased in control mice $1 \mathrm{~h}$ after exposure to both mild or restraint stress, likely resulting from activation of negative feedback circuits through limbic pathways and directly through the PVN. CRH expression did not decrease in the PVN in FBGRKO mice, indicating that sustained forebrain drive is able to counteract direct effects of glucocorticoids on the PVN to regulate CRH expression.

MR is an important regulator of HPA axis function, and deletion of this receptor leads to increased plasma CORT concentrations (Gass et al., 2000, 2001). MR expression is equivalent in FBGRKO and control mice in nonstressed conditions and after a mild stressor. A more severe stress, restraint stress, led to a significant decrease in the expression of MR in area CA1 and the dentate gyrus of the hippocampus of FBGRKO mice. These data suggest that, although the loss of forebrain GR signaling can lead to changes in regulation of MR expression, these changes are subtle and unlikely to compensate for the loss of forebrain GR.

Glucocorticoids downregulate the expression of CRHR1 mRNA in the PVN, although effects in the hippocampus have not been described (Makino et al., 1995). The loss of forebrain GR signaling led to increased CRHR1 mRNA in the hippocampus of FBGRKO mice, consistent with the pattern observed for CRHR1 regulation in the PVN. This receptor has been strongly implicated in the regulation of anxiety, and CRHR1 antagonists are known to have both anxiolytic and antidepressant properties (Muller et al., 2003). The increase in CRHR1 mRNA in FBGRKO mice supports the notion that the behaviors observed in the FBGRKO mice could reflect increased agitation rather than decreased anxiety-like behavior.

In this study, we demonstrate that a primary defect in forebrain GR signaling, in regions encompassing the cerebral cortex, hippocampus, nucleus accumbens, caudate-putamen, basolateral and basomedial amygdala, and bed nucleus of the stria terminalis, leads to alterations in behavioral and physiological reactivity to stress. These changes likely reflect increased agitation or impulsivity and an exaggerated fight-or-flight response in FBGRKO mice. In addition, we show that the observed anxiety-like phenotype is not reversed after chronic treatment with the antidepressant imipramine, unlike despair-related behaviors, which are reversed after similar antidepressant treatment (Boyle et al., 2005). Thus, although forebrain GR are involved in regulation of both anxiety- and despair-related behaviors, different signaling pathways or circuits modulate these responses.

\section{References}

Barlow DH, Campbell LA (2000) Mixed anxiety-depression and its implications for models of mood and anxiety disorders. Compr Psychiatry 41:55-60.

Belzer K, Schneier FR (2004) Comorbidity of anxiety and depressive disorders: issues in conceptualization, assessment, and treatment. J Psychiatr Pract 10:296-306.

Bjorntorp P, Rosmond R (2000) The metabolic syndrome-a neuroendocrine disorder? Br J Nutr 83 [Suppl 1]:S49-S57.

Boyle MP, Brewer JA, Funatsu M, Wozniak DF, Tsien JZ, Izumi Y, Muglia LJ (2005) Acquired deficit of forebrain glucocorticoid receptor produces depression-like changes in adrenal axis regulation and behavior. Proc Natl Acad Sci USA 102:473-478.

Brewer JA, Khor B, Vogt SK, Muglia LM, Fujiwara H, Haegele KE, Sleckman BP, Muglia LJ (2003) T-cell glucocorticoid receptor is required to suppress COX-2-mediated lethal immune activation. Nat Med 9:1318-1322.

Chrousos GP (2000) The role of stress and the hypothalamic-pituitaryadrenal axis in the pathogenesis of the metabolic syndrome: neuroendocrine and target tissue-related causes. Int J Obes Relat Metab Disord 24 [Suppl 2]:S50-S55.

Coryell WH, Black DW, Kelly MW, Jr Noyes R (1989) HPA axis disturbance in obsessive-compulsive disorder. Psychiatry Res 30:243-251.

De Kloet ER, Vreugdenhil E, Oitzl MS, Joels M (1998) Brain corticosteroid receptor balance in health and disease. Endocr Rev 19:269-301.

DeRijk R, Sternberg EM (1997) Corticosteroid resistance and disease. Ann Med 29:79-82.

Ehlert U, Gaab J, Heinrichs M (2001) Psychoneuroendocrinological contributions to the etiology of depression, posttraumatic stress disorder, and stress-related bodily disorders: the role of the hypothalamus-pituitaryadrenal axis. Biol Psychol 57:141-152.

Gass P, Kretz O, Wolfer DP, Berger S, Tronche F, Reichardt HM, Kellendonk C, Lipp HP, Schmid W, Schutz G (2000) Genetic disruption of mineralocorticoid receptor leads to impaired neurogenesis and granule cell degeneration in the hippocampus of adult mice. EMBO Rep 1:447-451.

Gass P, Reichardt HM, Strekalova T, Henn F, Tronche F (2001) Mice with targeted mutations of glucocorticoid and mineralocorticoid receptors: models for depression and anxiety? Physiol Behav 73:811-825.

Gorman JM (1996) Comorbid depression and anxiety spectrum disorders. Depress Anxiety 4:160-168.

Gorwood P (2004) Generalized anxiety disorder and major depressive disorder comorbidity: an example of genetic pleiotropy? Eur Psychiatry 19:27-33.

Herman JP, Figueiredo H, Mueller NK, Ulrich-Lai Y, Ostrander MM, Choi DC, Cullinan WE (2003) Central mechanisms of stress integration: hierarchical circuitry controlling hypothalamo-pituitary-adrenocortical responsiveness. Front Neuroendocrinol 24:151-180.

Holsboer F (1999) The rationale for corticotropin-releasing hormone receptor (CRH-R) antagonists to treat depression and anxiety. J Psychiatr Res 33:181-214.

Holsboer F (2000) The corticosteroid receptor hypothesis of depression. Neuropsychopharmacology 23:477-501. 
Invitti C, Redaelli G, Baldi G, Cavagnini F (1999) Glucocorticoid receptors in anorexia nervosa and Cushing's disease. Biol Psychiatry 45:1467-1471.

Kellendonk C, Gass P, Kretz O, Schutz G, Tronche F (2002) Corticosteroid receptors in the brain: gene targeting studies. Brain Res Bull 57:73-83.

Laugero KD (2001) A new perspective on glucocorticoid feedback: relation to stress, carbohydrate feeding and feeling better. J Neuroendocrinol 13:827-835.

Makino S, Schulkin J, Smith MA, Pacak K, Palkovits M, Gold PW (1995) Regulation of corticotropin-releasing hormone receptor messenger ribonucleic acid in the rat brain and pituitary by glucocorticoids and stress. Endocrinology 136:4517-4525.

Muller MB, Zimmermann S, Sillaber I, Hagemeyer TP, Deussing JM, Timpl P, Kormann MS, Droste SK, Kuhn R, Reul JM, Holsboer F, Wurst W (2003) Limbic corticotropin-releasing hormone receptor 1 mediates anxiety-related behavior and hormonal adaptation to stress. Nat Neurosci 6:1100-1107.
Oitzl MS, van Haarst AD, Sutanto W, de Kloet ER (1995) Corticosterone, brain mineralocorticoid receptors (MRs) and the activity of the hypothalamic-pituitary-adrenal (HPA) axis: the Lewis rat as an example of increased central MR capacity and a hyporesponsive HPA axis. Psychoneuroendocrinology 20:655-675.

Schaefer ML, Wong ST, Wozniak DF, Muglia LM, Liauw JA, Zhuo M, Nardi A, Hartmann RE, Vogt SK, Luedke CE, Storm DR, Muglia LJ (2000) Altered stress-induced anxiety in adenylyl cyclase type VIII-deficient mice. J Neurosci 20:4809-4820.

Tollefson GD, Souetre E, Thomander L, Potvin JH (1993) Comorbid anxious signs and symptoms in major depression: impact on functional work capacity and comparative treatment outcomes. Int Clin Psychopharmacol 8:281-293.

Tsien JZ, Chen DF, Gerber D, Tom C, Mercer EH, Anderson DJ, Mayford M, Kandel ER, Tonegawa S (1996) Subregion- and cell type-restricted gene knockout in mouse brain. Cell 87:1317-1326. 